\title{
Medicine misuse: a systematic review and proposed hierarchical terminology
}

\author{
Allison Singier ${ }^{1}$, Pernelle Noize ${ }^{1}$, Driss Berdai ${ }^{2}$, Amelie Daveluy $^{2}$, Mickael Arnaud $^{1}$, \\ mathieu Molimard ${ }^{2}$, Bernard Begaud ${ }^{1}$, and Francesco Salvo ${ }^{1}$ \\ ${ }^{1}$ University of Bordeaux \\ ${ }^{2} \mathrm{CHU}$ de Bordeaux Service de Pharmacologie Medicale
}

June 10, 2020

\begin{abstract}
Aim: Although medicine misuse is a public health issue, it has multiple meanings in the medical literature. This study aimed to characterize, classify and identify the most appropriate definitions of medicine misuse. Methods: A systematic review was performed in Medline, ISI Web of Science, SocINDEX, PsycInfo, PsycArticles, and Psychological and Behavioral Sciences Collection, using keywords related to misuse, appropriateness, and medicine between November 1st, 2008 and November 1st, 2018. Additional searches were conducted in websites of regulatory agencies and public health institutions. Two authors independently selected studies providing both definitions and examples of misuse, while a third resolved disagreements. Definitions were used to propose a hierarchical classification based on initiator, intent, purpose, and context of medicine misuse. The study is registered on PROSPERO: CRD42018115789. Results: Of 2,901 identified records, 44 were included. A total of 63 definitions and 60 examples of misuse were retrieved. When the prescriber is initiator and according to intent, potential medicine misuse referred to "intentional or unintentional prescribing not in line with clinical evidence". Based on context, he could prescribe medicines not clinically justified, i.e. "overprescribing", or prescribe indicated medicines incorrectly, i.e. "misprescribing". Among other groups of definitions, those overlapping with drug abuse or medication use errors were considered out-of-scope. Conclusion: This systematic review provides a comprehensive overview of the terms and definitions used to characterize medicine misuse and could serve as a basis for a terminology that makes clear distinctions between misuse, abuse, and errors.
\end{abstract}

\section{Hosted file}

Singier_Manuscript_BJCP.doc available at https://authorea.com/users/331912/articles/458407medicine-misuse-a-systematic-review-and-proposed-hierarchical-terminology 


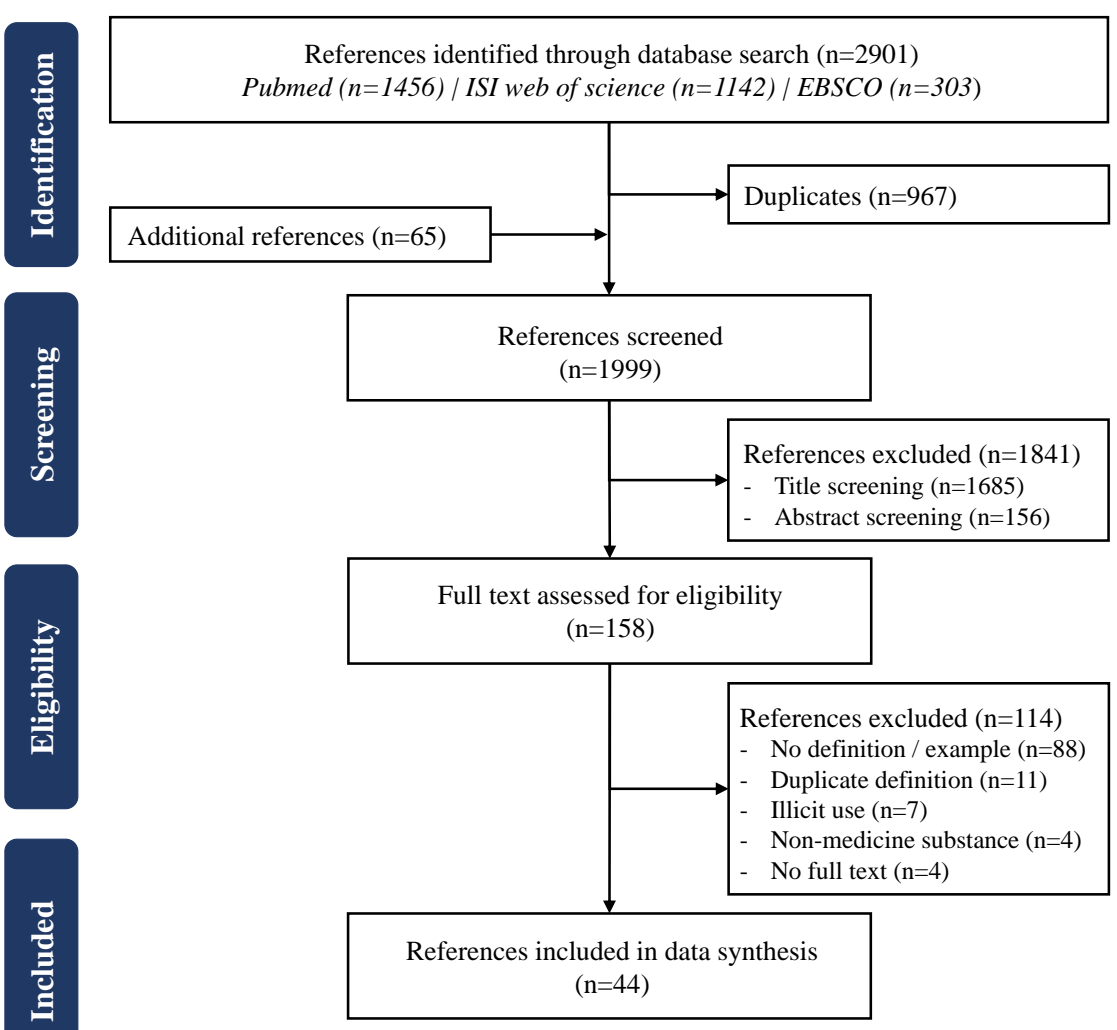




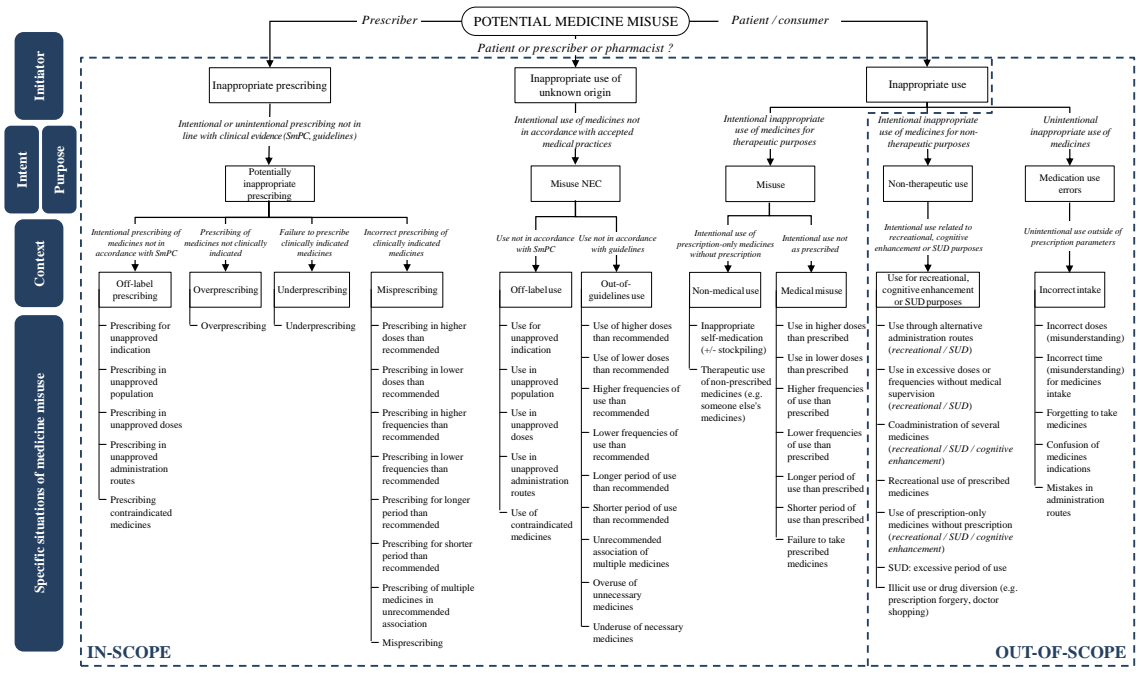

$\square$ Terms of interest: from most general at top to most precise at bottom $\quad-$ Italic $\rightarrow$ Meanings of terms of interest $\quad-$ list List of situations associated with the above tems 